\title{
Compact Thomson parabola spectrometer for fast diagnostics of different intensity laser-generated plasmas
}

\author{
L. Torrisi* and G. Costa \\ Dipartimento di Scienze Fisiche, MIFT, Università di Messina, \\ V.le F.S. D’Alcontres 31, 98166 San Agata, Messina, Italy
}

(Received 21 November 2018; published 25 April 2019)

\begin{abstract}
A compact Thomson parabola spectrometer for diagnostics of laser-generated plasma, projected in Messina University, has been employed in different experiments concerning diagnostics of lasergenerated plasmas. It allows to detect charged particles emitted from hot laser plasma and fast analyzing of their charge state, kinetic energy and mass-to-charge ratio. Moreover, it is possible to detect electrons emitted from laser-generated plasma. The spectrometer consists of a double pinhole input for alignment direction, a permanent magnet $(0.004-4 \mathrm{kG})$ and an electric field $(0.05-5 \mathrm{kV} / \mathrm{cm})$ both orthogonal to the direction of the incident particles, and different type of planar detectors (multichannel plates, phosphorous screen, gafchromic, CR39 and PM 355 track detectors). Measurements have been acquired at MIFT in Messina observing electrons up to $10 \mathrm{keV}$ kinetic energy, at INFN-LNS of Catania using ions emitted from plasma submitted to a postacceleration up to $30 \mathrm{kV}$ per charge state and at PALS Laboratory in Prague detecting energetic ions above $1 \mathrm{MeV}$ per charge state. The particles recognition using the Thomson spectrometer has been obtained comparing the experimental parabola curves with the theoretical simulations obtained using COMSOL software. Results will be presented and discussed.
\end{abstract}

DOI: 10.1103/PhysRevAccelBeams.22.042902

\section{INTRODUCTION}

Ion streams generated by laser induced plasmas, with an energy of the order of $\mathrm{keV}-\mathrm{MeV}$, can be detected using different devices such as Faraday cups, semiconductor detectors, gafchromic films, track detectors, and others. The high energy of accelerated ions can be obtained using ns-fs laser pulse durations and lasers intensities ranging between $10^{10}$ and $10^{16} \mathrm{~W} / \mathrm{cm}^{2}[1,2]$. A high-intensity laser hitting a target causes a plasma production with the expulsion of high-energy electrons, ions and photons, with angular emission narrow for the particles and large for the photons. The ion acceleration regime depends on the laser properties, irradiation conditions and target characteristics.

At low laser intensities, the backward plasma acceleration (BPA) in thick targets occurs, while at high intensities the target normal sheath acceleration (TNSA) from thin targets can occur [3]. These two types of acceleration regimes permit to accelerate, respectively, high ion currents and low kinetic energies (BPA) or low ion currents and

\footnotetext{
*Corresponding author. ltorrisi@unime.it

Published by the American Physical Society under the terms of the Creative Commons Attribution 4.0 International license. Further distribution of this work must maintain attribution to the author(s) and the published article's title, journal citation, and DOI.
}

high energies (TNSA) [4], opening new frontiers to suggestive applications, such as laser ion sources, induction of nuclear reactions, compact electrons and x-ray generators, preparation of proton beams for radiotherapy, and many others [5].

With the aim to characterize the laser-produced plasma, especially to use it as an ion source and ion acceleration method, many types of spectrometers can be employed for the plasma diagnostics, such as optical measurements in visible (VIS), UV and x-ray wavelength regions to determine the temperature and electron plasma density. Fast streak cameras permit to measure the plasma expansion velocity and the temperature gradients during the expansion. Langmuir probes are employed to evaluate the plasma potential, temperature and density. The charged particles detection using fast semiconductors is a valid method to determine the ion and electron energy distributions. Mass spectrometers used for the particle mass emission evaluations permit to determine the ablation yield [6-8]. The Thomson parabola spectrometer (TPS) appears the most appropriated between other techniques thanks to its possibility to distinguish the ion mass, charge state and energy with high resolution. The TPS diagnostics permit to solve the recognition problems of the ion species (charge state and mass) and of energetic electrons. The device gives the possibility to evaluate quickly some parameters, such as the maximum energy of protons and ions, the amount of ion 
charge states for each species in the plasma, the type of different ions accelerated by laser-induced plasma, and the measure of medium and maximum electron energy, according to their magnetic and electric deflection. Moreover, by the signal intensity of the parabola curves, it is possible to evaluate the particle energy distribution for each ion species and their charge state distribution, according to the literature [9]. In addition, the use of TPS for electrons permits to have direct information on the energy of hot and cold electrons emitted from the nonequilibrium plasma.

The modern TPS, generally, has high sensitivity and precision but large dimensions (1-2 meters). The cost of these devices is high due to the use of expensive electromagnets that absorb high currents, to the high vacuum technology and to the use of microchannel plate (MCP) detectors coupled to phosphorous screen and fast CCD cameras [10].

In this paper, we propose the diagnostics of plasmas generated by high laser intensity using a compact (low volume), cheap and simple TPS. The volume compactness of this spectrometer is given in respect to the larger ones present at INFN-LNS in Catania [10], Prague Asterix Laser System (PALS) Laboratory in Prague [11] and other big facilities such as the Queen's University in Belfast [12].

Before the alignment pinholes, our TPS has a ring ion collector in input to control the flux of ions arriving in the direction of the TPS $\mathrm{z}$ axis and it uses permanent magnets and gafchromic detectors for the ions and electron parabola images.

For high ion energies (higher than $1 \mathrm{MeV}$ per charge state) the deflection must use high permanent magnetic fields, of the order of $3 \mathrm{kGauss}$, and electrical fields, of the order of $15 \mathrm{kV} / \mathrm{cm}$, while for low ion energies such fields are lower. The different parabolas of the ions recorded on the gafchromic detectors can be well separated spatially enhancing the distance between the deflection field and the detector distance. A TPS limitation is due to a maximum proton detection of about $20 \mathrm{MeV}$.

Of course, this simple spectrometer is less accurate in sensitivity and precision than those mentioned above because it does not use the MCP system but it is very useful for a very fast first diagnostic and for its transportability in each laboratory. The compact TPS has dimensions about a factor 2-5 times lower and a cost up to a factor 10 lower.

This spectrometer deflects the ions by a magnetic and electric field orthogonal to the direction motion of the charged particles, allowing information on the ion species, energy and charge state, as reported in recent literature $[13,14]$.

According to the Lorentz force and the deflection of charged particles from electric fields, if the particle moves along the $\mathrm{z}$ axis, the dependence of the electrical deflection (y direction) from the magnetic field directed along the $\mathrm{x}$ direction will take the form

$$
y=\frac{m}{q} \frac{E}{B^{2}} \frac{L_{E}}{L_{B}^{2}}\left(\frac{1}{2} L_{B}+d_{B}\right)^{-2}\left(\frac{1}{2} L_{E}+d_{E}\right) x^{2},
$$

where $\mathrm{m} / \mathrm{q}$ is the mass to charge ratio, $\mathrm{E}$ and $\mathrm{B}$ are respectively the electric and magnetic fields, $\mathrm{L}_{E}$ and $\mathrm{L}_{B}$ are respectively the lengths of the electric and magnetic fields, and $\mathrm{d}_{E}$ and $\mathrm{d}_{B}$ are respectively the distances between the electric and magnetic field positions and the final detector.

The entrance ring ion collector permits to probe the ion yield and average velocity, by using time-of-flight measurements, being placed at a known distance, $\mathrm{d}_{\mathrm{ICR}}$, from the target irradiated by the laser.

The advantage of this instrument derives by his compactness, of about $30 \mathrm{~cm}$ maximum length, use of permanent magnets instead of electromagnets and simple transportability between one laboratory and another. It can be used for plasma analysis produced by low and high laser intensity, uses different permanent magnets, with higher B for higher ion energy.

The results obtained with this compact TPS will be presented and discussed.

\section{MATERIAL AND METHODS}

The measurements presented in this paper were obtained in three different laboratories using the same compact TPS instrument. A schematization of the three experimental setups is reported in Fig. 1(a), while a picture of this is shown in Fig. 1(b). The laser pulse interacts with a solid target with an incidence angle of $45^{\circ}$ placed in a high vacuum chamber. The produced plasma, developed along the normal to the target surface ( $\mathrm{z}$ direction), is directed towards an electromagnetic TPS spectrometer analyzing the mass-to-charge $(\mathrm{m} / \mathrm{q})$ ratio. For TNSA accelerations the foil is very thin (microns), the laser arrives from the back of the target and the plasma is produced in the forward direction. For backward plasma acceleration (BPA) the laser incidence occurs on the surface from which will be emitted the plasma and the target generally is thick $(\sim 1 \mathrm{~mm})$.

A first experiment has been performed at INFN-LNS (South National Laboratories) in Catania, in collaboration with the MIFT Department of the Messina University, using a thick target in BPA regime, which can be positively polarized to induce Coulomb repulsion and post ion acceleration in the backward direction, as reported in the literature [15].

In this case, an Nd:YAG laser, with a fundamental wavelength of $1064 \mathrm{~nm}$, a maximum pulse duration of $9 \mathrm{~ns}$, and variable energy between 1 and $900 \mathrm{~mJ}$, is focused on a target, consisting of titanium oxide, through an optical lens of focal length $50 \mathrm{~cm}$.

The irradiation occurs in high vacuum at $10^{-7} \mathrm{mbar}$. The generated plasma emits ions with low energy (energy of the order of $200 \mathrm{eV}$ per charge state), which is not enough for 

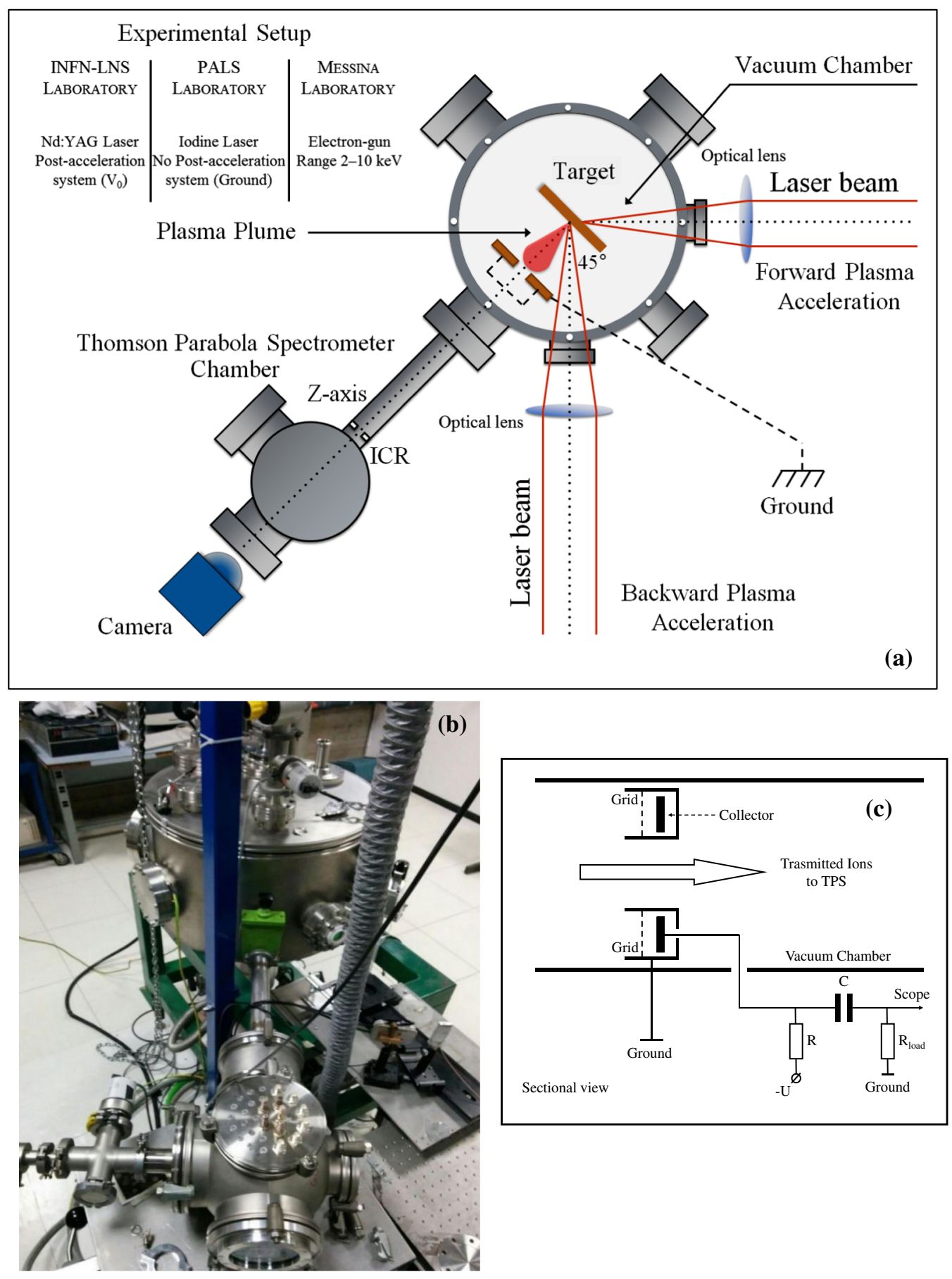

FIG. 1. Schematization of experimental setup using laser irradiations in TNSA and BPA regimes and plasma production directed towards the TPS spectrometer (a), photos of the external camera (top view) (b) and scheme of the used ICR (c).

an optimal TPS deflection, so ions are submitted to a postacceleration system to be further accelerated.

The target holder can be polarized with a potential ranging from 0 to $60 \mathrm{kV}$ positive, so the ions produced by the laser ablation are rejected and accelerated towards the ground potential at energies that can reach up to $60 \mathrm{keV}$ per charge state.

The obtained plasma is previously analyzed by an ICR (ion collector ring), with and without postacceleration, using a time-of-flight (TOF) technique. In addition, the produced ion beam is collimated by two aligned pinholes along the $\mathrm{z}$ axis of the TPS to be analyzed in detail.

The ICR consists in an annular Faraday cup with a secondary electron suppression polarized to $-100 \mathrm{~V}$, whose collector is coupled to a fast storage oscilloscope through a small capacitor, as reported in the scheme of Fig. 1(c). Both ICR and TPS are aligned along the $\mathrm{z}$ axis and are assembled in a vacuum chamber whose photo is reported in Fig. 1(b). 

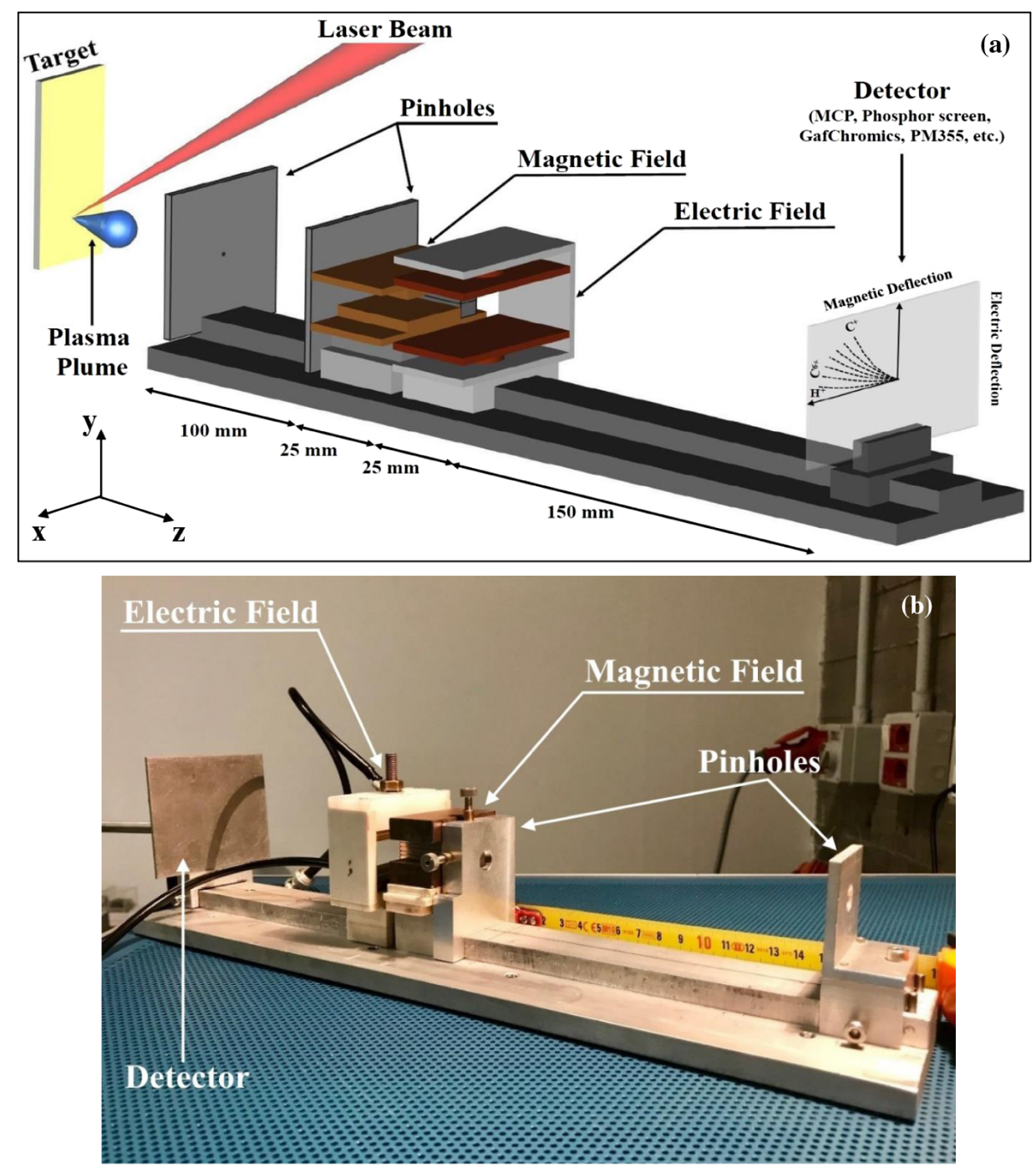

FIG. 2. Schematization and photos of the Thomson parabola spectrometer.

A second experiment has been performed at PALS Laboratory in Prague using a thin foil irradiated in the TNSA regime to induce forward ion acceleration. In this case, an iodine laser was employed to irradiate a polyethylene foil, such a thick (1 mm) and thin (10 microns) thickness target.

The laser has a fundamental wavelength of $1315 \mathrm{~nm}$, a maximum pulse duration of $300 \mathrm{ps,}$ pulse energy up to $700 \mathrm{~J}$ and a focal spot diameter of $70 \mu \mathrm{m}$. In this case, the plasma ion stream is produced at high kinetic energy, of the order of some $\mathrm{MeV}$ per charge state. The plasma was analyzed both with ICR-TOF and with TPS spectrometers.

A third experiment was performed at Messina Laboratory, using a setup similar to that reported in Fig. 1(a), but with an electron gun placed in the vacuum chamber, which emits electrons along the $\mathrm{z}$ axis and directed towards the ICR and TPS spectrometer. The produced electrons have high current $(\mu \mathrm{A})$, and kinetic energy ranging between 2 and $10 \mathrm{keV}$.
The TPS used for these three measurements is the same compact reported in the schematization of Fig. 2(a), of which a photo is reported in Fig. 2(b). It is constituted by two input pinholes, to guarantee the directivity of the incident ions, distant from each other $100 \mathrm{~mm}$, and with holes of $1 \mathrm{~mm}$ (input) and $100 \mu \mathrm{m}$ (output) in diameter respectively. Immediately afterwards, there is a magnetic and an electric deflection field, both $25 \mathrm{~mm}$ long. The fields are directed parallel to each other, and orthogonal to the direction of motion of the incident particles. Finally, at a distance of $150 \mathrm{~mm}$ from the electric field, there is a planar detector, which can employ different recording methods. The TPS system is placed in a high vacuum at $10^{-7} \mathrm{mbar}$ [Fig. 1(b)].

The magnetic and electric fields used for the experimental setups, prepared at INFN-LNS Catania Laboratory and at PALS in Prague are the same, and correspond to 2500 Gauss and $0.67 \mathrm{kV} / \mathrm{cm}$, respectively. In such experiments, the detector used for TPS was a gafchromic film and 


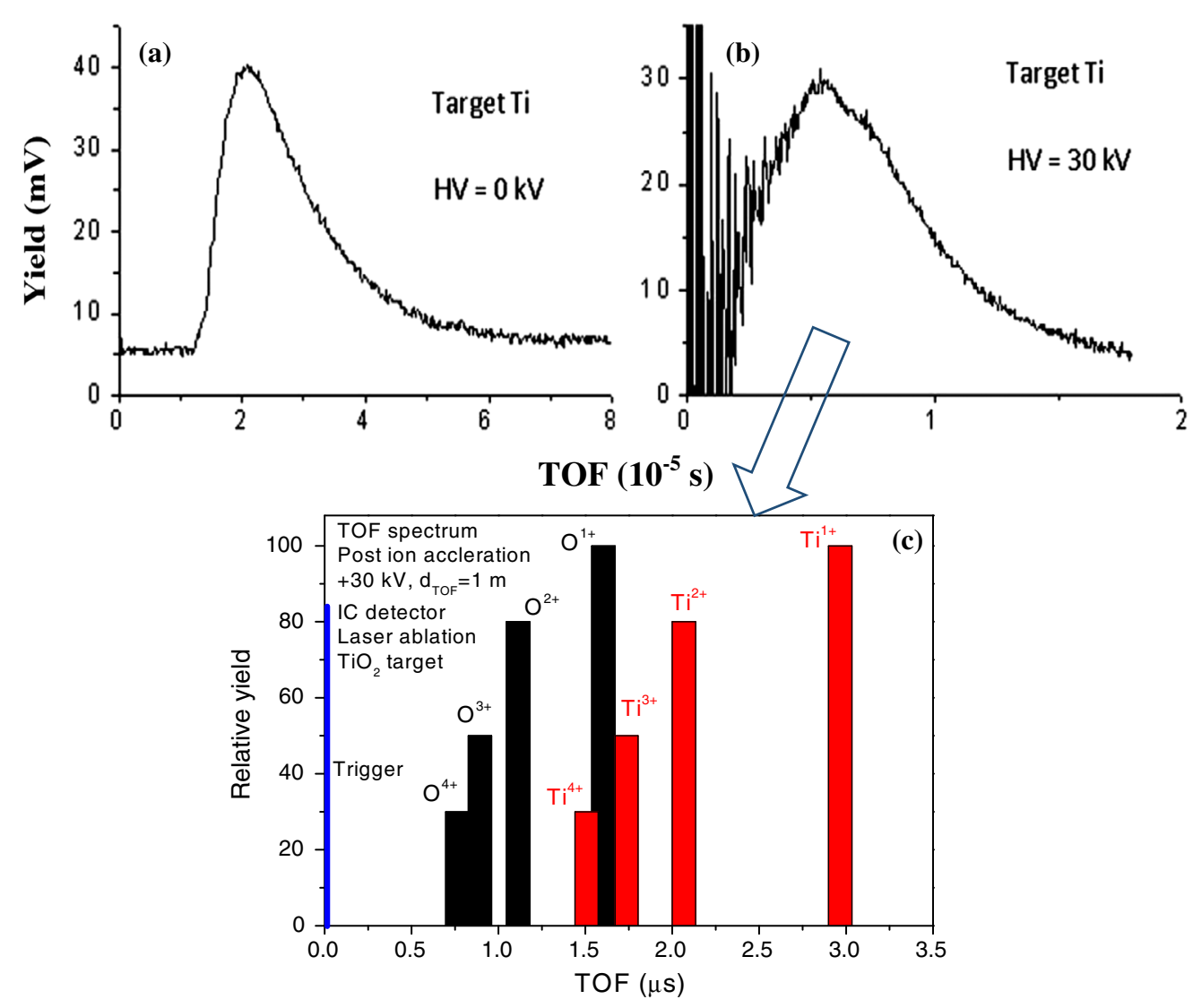

FIG. 3. TOF spectra acquired by the ICR detector, when the potential applied to postacceleration system is $0 \mathrm{~V}$ (a) and $30 \mathrm{kV}$ (b), by irradiating titanium oxide target, and ion deconvolution elaboration (c) [15].

a PM355 track detector [16]. The track detector was chemical etched as a function of the solution concentration, temperature and time, to reveal the most superficial heavy ion tracks or the deepest tracks due to energetic protons.

The measurements performed at Messina Laboratory (University of Messina) have employed the same TPS with a magnetic and electric field of 7.5 Gauss and $0.1 \mathrm{kV} / \mathrm{cm}$ respectively. In such a case, the TPS detector for the electron beam measurements was a luminescent phosphor screen.

COMSOL Multiphysics software was employed to perform some simulations of the produced ions from the plasma source, of their deflection applying the magnetic and electric fields [17]. The COMSOL Multiphysics simulation software environment facilitates all steps in the modeling process defining the geometry, meshing, specifying the involved physics mechanisms, solving, and then visualizing the results [18].

\section{RESULTS AND DISCUSSION}

By irradiating a titanium oxide target with the Nd:YAG laser of the INFN-LNS Laboratory in Catania, two IC timeof-flight spectra (TOF) were obtained, when the postacceleration potential is absent $(0 \mathrm{~V})$ and present $(30 \mathrm{kV})$; these results are reported in Fig. 3 [15].
The IC spectra are triggered with the laser shot at $\mathrm{TOF}=0$ time. The TOF spectrum obtained without the postacceleration system [Fig. 3(a)] represents the convolution of all detected ion species, and indicates that the faster ions (protons) are positioned at a time of $7 \mu$ s [protons as impurities are not very evident in the used scale of Fig. 3(a)]. Since the target distance from the ICR is $100 \mathrm{~cm}$, these will have a maximum kinetic energy of about $106 \mathrm{eV}$. The oxygen ions have a minimum TOF of about $13 \mu \mathrm{s}$, corresponding to a maximum energy of about $492 \mathrm{eV}$ and the titanium ion contribution is detected at a TOF of about $22 \mu \mathrm{s}$, corresponding to a maximum kinetic energy of about $516 \mathrm{eV}$. These results indicate that the plasma ion acceleration is of about $106 \mathrm{eV}$ per charge state and that the maximum charge states of the $\mathrm{O}$ and $\mathrm{Ti}$ ions are $4+$. The ionization potentials of $\mathrm{O}^{4+}$ and $\mathrm{Ti}^{4+}$ are 77.4 and $43.3 \mathrm{eV}$, thus the maximum electron energy, comparable to the plasma temperature, should be lower than $100 \mathrm{eV}$.

When a postaccelerative potential of $30 \mathrm{kV}$ is applied between target and ground, the obtained TOF spectrum is that shown in Fig. 3(b). In this case, the arrival time of the ions decreases significantly because ions are postaccelerated to $30 \mathrm{keV}$ per charge state. The TOF peaks of $\mathrm{O}$ ions are placed at $1.67,1.18,0.96$, and $0.83 \mu \mathrm{s}$, for $\mathrm{O}^{1+}, \mathrm{O}^{2+}$, $\mathrm{O}^{3+}$, and $\mathrm{O}^{4+}$, respectively. The TOF peaks of $\mathrm{Ti}$ ions are 


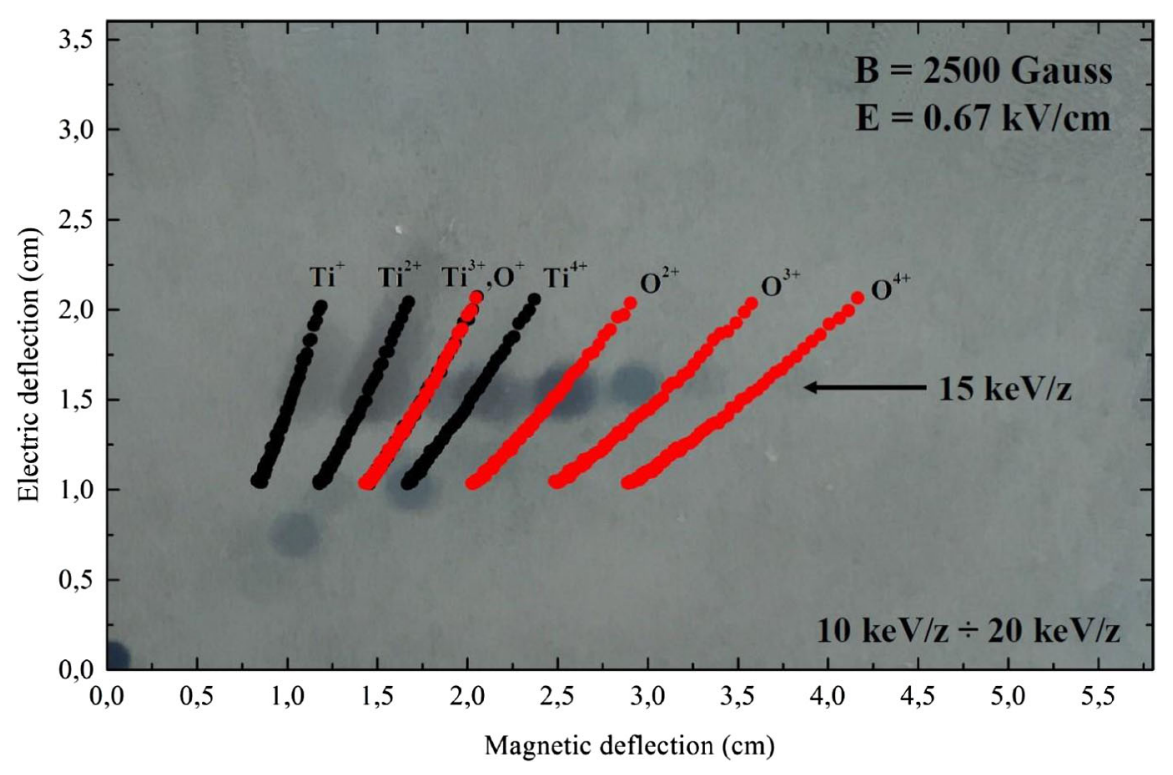

FIG. 4. Comparison of spectrogram of titanium and oxygen ions obtained through laser irradiation in repetition rate mode, using a $15 \mathrm{kV}$ postaccelerator potential, and simulation data obtained by COMSOL.

placed at 2.9, 2.0, 1.67, and $1.44 \mu \mathrm{s}$, for $\mathrm{Ti}^{1+}, \mathrm{Ti}^{2+}, \mathrm{Ti}^{3+}$, and $\mathrm{Ti}^{4+}$, respectively, as reported in the ion deconvolution elaboration of the IC spectrum of Fig. 3(c).

Results confirm the expected values of $30 \mathrm{keV}$ for both main ions. Less evident by ICR but more evident using the TPS spectrometer, it is possible to distinguish the energies of $30,60,90$ and $120 \mathrm{keV}$, due to the acceleration of the charge states $1+, 2+, 3+$ and $4+$, respectively [15].

ICR does not detect very well all the ions emitted from plasma because its detection solid angle is very limited (about $0.2 \mathrm{msr}$ ), its position is not along the normal to the target surface ( $\mathrm{z}$ axis), and it is influenced from the fast and intense electromagnetic fields produced by the laser shotgenerating plasma [19].

After the preliminary analysis performed using the ICR detector, the postaccelerated ions are analyzed with a TPS deflection system. The presented measurements were performed using a postacceleration potential settled to $15 \mathrm{kV}$, so the ions acquire $15 \mathrm{keV}$ per charge state. Figure 4 shows the spectrogram acquired using the gafchromic film (HD-810 dosimeter [20]) as a detector placed in the TPS and a laser operating at $1 \mathrm{~Hz}$ repetition rate mode. In this experiment, the electric and magnetic field were of $0.67 \mathrm{kV} / \mathrm{cm}$ and 2500 Gauss, respectively.

Through the comparison of experimental parabolas and the simulation data calculated by COMSOL Multiphysics software, which is able to reconstruct the trajectory of the ions in the system of interest, it is possible to recognize the energies and charge states of each charged species in the plasma. Ion beams with energies between 10 and $20 \mathrm{keV}$ per charge state were simulated. The overlapping of the experimental and simulation data reported in Fig. 4 showed an excellent agreement. In this particular case, the energy is fixed due to the postacceleration system, the comparison between the experimental and simulation data makes it possible to identify the species that make up the studied plasma.

Results confirm the four charge states for titanium and for oxygen ions, as expected. Because of the low dose provided by the protons, present only as an impurity in the target, it is not possible to impress them on the device. In fact, due to heating effects on the target, and removal of material from it, after the first two-three laser shots the protons can no longer be observed appreciably.

Another application of our TPS spectrometer was performed to analyze the ions produced by a plasma generated irradiating a polyethylene (PE) thin target in the TNSA regime at $10^{16} \mathrm{~W} / \mathrm{cm}^{2}$ intensity at PALS Laboratory in Prague. Figure 5(a) reports the ICR spectrum at $80 \mathrm{~cm}$ distance from the irradiated PE target. The spectrum shows a high photopeak due to the detection of $\mathrm{x}$ rays on the collector, a little peak at $50 \mathrm{~ns}$ due to fast protons at a kinetic energy of $1.3 \mathrm{MeV}$ and a large and intense peak due to the different contribution of carbon peak, from $\mathrm{C}^{1+}$ (slower) up to $\mathrm{C}^{6+}$ (faster). Moreover, a long tail demonstrates that also slowly ions are detected, due detection of $\mathrm{C}_{x} \mathrm{H}_{y}$ molecular groups, according to the literature [21]. The presented deconvolution of $\mathrm{H}$ and $\mathrm{C}$ ion peaks are obtained on the base of the Coulomb-Boltzmannshifted distribution function [2], assuming the proton energy as representative of the value of the light ion acceleration per charge state. It means that the $\mathrm{C}^{1+}$ has the same energy of the protons and that the $\mathrm{C}^{6+}$ ion energy is about $1.3 \times 6=7.8 \mathrm{MeV}$, on the peak. The ion energy distributions are Boltzmann curves shifted proportionally to the ion charge state. The theoretical approach permits to 

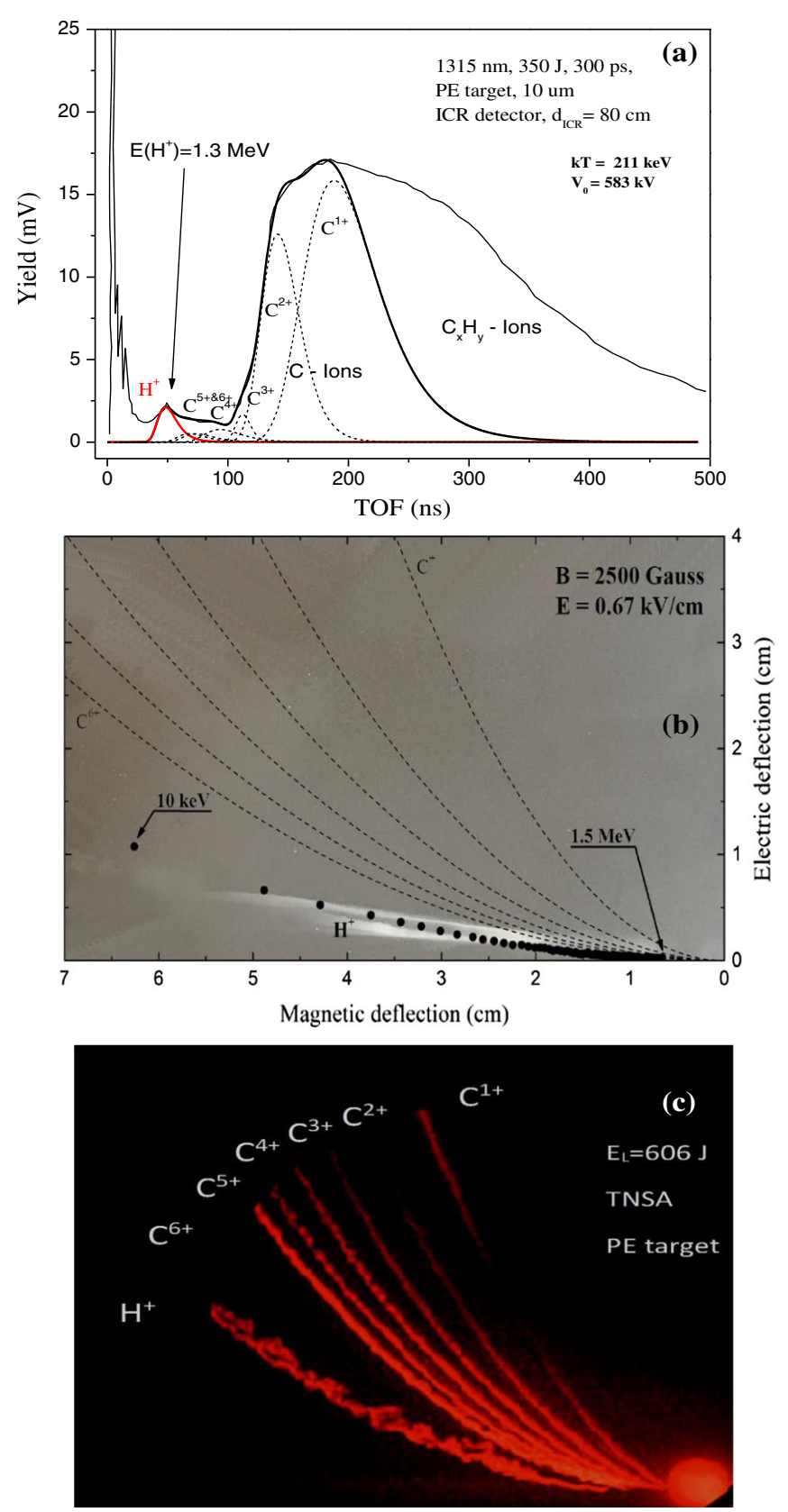

FIG. 5. ICR fit-deconvolution spectrum (a), comparison between TPS experimentally parabola spectrum with data by COMSOL simulation (b), and TPS spectrum using MCP (c) of polyethylene (PE) obtained with $10^{16} \mathrm{~W} / \mathrm{cm}^{2}$ intensity at PALS Laboratory in Prague.

evaluate a plasma temperature of about $211 \mathrm{keV}$ and an acceleration potential of about $583 \mathrm{kV}$.

Figure 5(b) shows the overlap between the experimental TPS spectrum and the simulation of the ionic traces, obtained with COMSOL Multiphysics. In this case, the ions are recorded using a PM355 track detector and the electric and magnetic field used were the same of the previous experiment $(0.67 \mathrm{kV} / \mathrm{cm}$ and 2500 Gauss, as previously described). To read these track films it is necessary to perform a suitable chemical etching controlling solution $(\mathrm{NaOH})$ concentration, time duration and etching temperature.

Depending on the duration of the processing, it is possible to observe only the superficial traces of the carbon ions or protons at greater depth. The $1 \mathrm{MeV}$ protons, in fact, are implanted in the plastic detector at greater depths (25 microns); on the other hand, 1 and $6 \mathrm{MeV}$ carbon ions are implanted in the plastic detector at 2.5 and 8.5 microns, respectively, as calculated from SRIM code [22]. Thus, when etching is obtained for quite long times the traces of carbon ions are erased, while only those of the protons emerge. Conversely, for a short time, the traces of carbon ions are visualized, while those of the protons remain hidden. In the presented data reported in Fig. 5(b) the traces of only protons are reported. The data allows observing a great agreement between the experimental data and those obtained by simulations. In this case, we were able to obtain the maximum value of energy observed for the protons by measuring the minimum distance between the zero deflection and the proton parabola points, which is near to $1.5 \mathrm{MeV}$, also confirmed by complementary measures. The carbon ion curves obtained by a theoretical calculation are shown in Fig. 5(b). Thus, the superficial etching has removed immediately the $\mathrm{C}$ ion tracks leaving the traces of the protons in the polymeric detector avoidable.

Figure 5(c) reports the TPS spectrum obtained using an MCP detector coupled with a red phosphorus screen and a CCD camera, irradiating at PALS a thin polyethylene foil (5 microns) irradiated in the TNSA regime using $606 \mathrm{~J}$ laser pulse energy. In this case, the TPS sensitivity is very high and the six states of charge of the carbon and the protons emitted by the plasma can be well separated. The maximum proton energy corresponds to $1.5 \mathrm{MeV}$.

Finally, we have used this TPS at the Messina MIFT Laboratory, for its calibration with $\mathrm{keV}$ electron beam detection. Figure 6 shows the photo of the spectrogram for electrons obtained experimentally and its overlap with the COMSOL simulation data.

The study of the latter case is important for the calibration of TPS in the detection of electrons coming from lasergenerated plasmas. Electron energy, in fact, is correlated to the plasma electron temperature and to the ion acceleration process in the TNSA regime. Due to their low energy of emission from the used electron gun, and for their very small mass, the magnetic and electric fields are significantly reduced with respect to previous cases. The experimental parabola of electrons is reported digitally, and superimposed on the curve obtained from the simulation of the electrons in the system of interest, by COMSOL software. The overlap between the experimental and simulative data shows a great agreement. This study has permitted the TPS calibration for electron beams with energies going from about 0.5 to $10 \mathrm{keV}$, representing a useful method to evaluate the electron plasma temperature of hot ionized gases. 

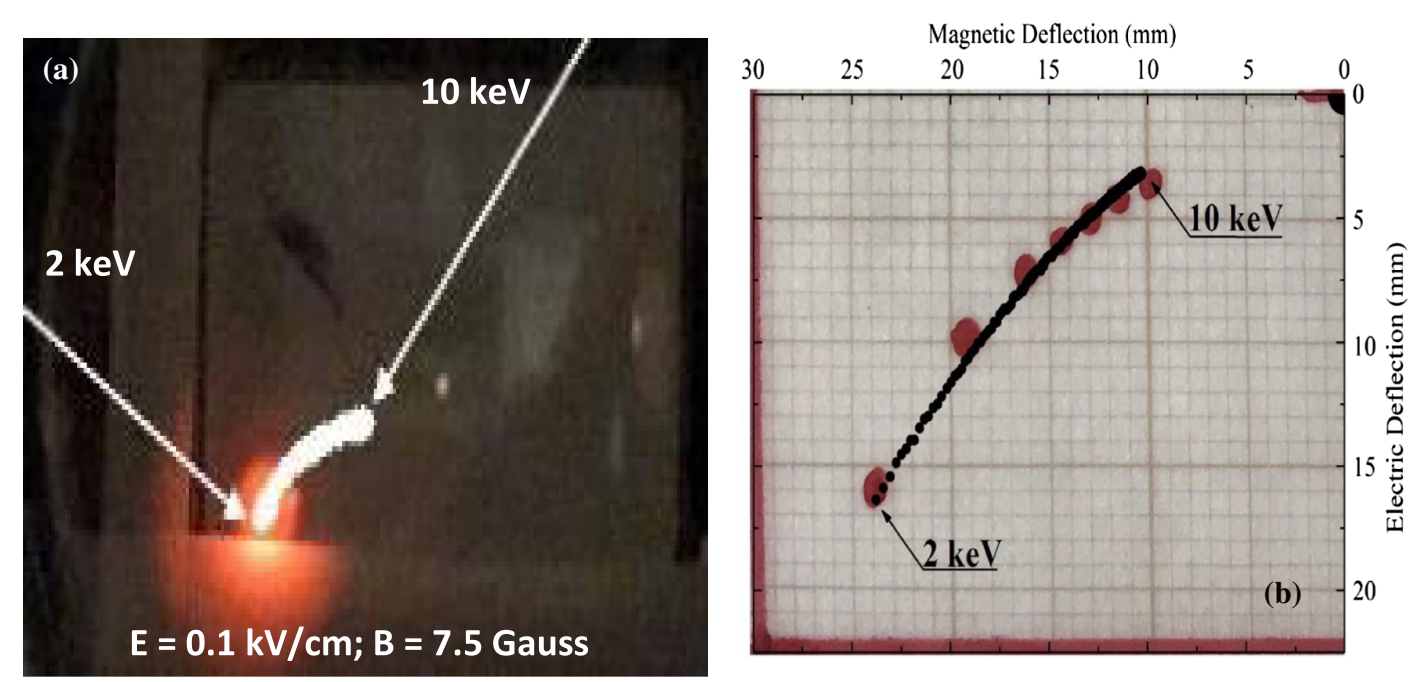

FIG. 6. Picture of the spectrogram obtained experimentally at the Messina Laboratories, using an electron gun (a), and overlapping with the simulation data obtained by COMSOL (b).

\section{CONCLUSIONS}

Many magnetic and electrical spectrometers can be used in the diagnostics of particles accelerated in plasmas by high-intensity laser pulses. For instance, the ion energy analyzer using electric deflection, the magnetic spectrometer chamber using magnetic deflection, and the complex mass spectrometers using quadrupole deflection reported in the literature $[23,24]$.

The presented compact TPS has the advantages to be a device portable and low volume, using permanent magnets and implemented with an ICR diagnostics for preliminary measurements of the maximum speed of ionic species, very useful for the characterization of ions and electrons coming from laser-generated plasma. The main advantages with respect to more expensive and larger TPS of big facilities consist of the simple construction, absence of high-current generators for electromagnets and expensive MCPs (can be used only gafchromic and ion track detectors).

It permits to evaluate the $m / q$ ratio of the particles analyzed. Knowing the mass of the ion, it is possible to know the degree of ionization of a plasma generated by laser pulses. In addition, the intensity of the curves impressed on the final detector can give information on the abundance of ions in the plasma, and therefore on the energy distributions of these. The presented TPS is a very compact device (length of about $30 \mathrm{~cm}$ and weight less than $5 \mathrm{~kg}$ ) that can be easily transported and that can be used both for cold and hot plasmas, generated by low and high laser intensities.

However, the compactness volume reduces the performance of the spectrometer because the limited magnetic and electrical deflection do not permit a better separation between many parabolas due to many charge states and ion species present in complex plasmas, and to measure very well high energetic particles, of the order of $10 \mathrm{MeV}$ per charge state.
Moreover, another defect in TPS could be that of failing to detect low-energy particles, such as those produced by medium-low intensity lasers $\left(<10^{14} \mathrm{~W} / \mathrm{cm}^{2}\right)$ accelerating ions at low energies of the order of $1 \mathrm{keV}$ per charge state or less [25]. In addition, the accuracy of the measure is affected by more errors, of the order of $15 \%$, due to the use of the less spatial resolution of gafchromic images with respect to the MCP equipment. This device, on the other hand, is very versatile and easy to read when applied to plasmas generated by high laser intensities both for backward BPA and forward TNSA regimes. In fact, the TPS gives a rapid response on the properties and species that make up a plasma characterized by energies of the order of tens of $\mathrm{MeV}$ by the state of charge or higher. A significant improvement can be obtained using a suitable $\mathrm{MCP}$ coupled to a phosphorous screen with a final CCD camera to obtain high spatial resolution parabola images using low and high ion energies, as that used at PALS Laboratory in the past years, of which one of our TPS spectrums is reported in Fig. 5(c) [21].

In this paper, we have shown how a compact (low volume) and portable TPS, cheap and simple to be built, can be used for the analysis and characterization of lasergenerated plasmas. The instrument can detect ions with energy ranging from tens of $\mathrm{keV}$ per charge state up to some $\mathrm{MeV}$ per charge state. We have also demonstrated the validity of the instrument and its reading speed, which gives detailed information on the properties of the plasma under examination.

Subsequently, we have used this device for its calibration to detect electrons from about $0.5 \mathrm{keV}$ up to $10 \mathrm{keV}$. Electrons of such energies are typical of hot plasmas, generated by high laser intensities, higher than $10^{14} \mathrm{~W} / \mathrm{cm}^{2}$. A fundamental aspect is the study of the electronic component of a nonequilibrium plasma generated by high-intensity pulsed lasers. In fact, the analysis of these electrons gives important 
information on temperature, density and charge states, and other properties of special interest of a laser generated plasma [26].

Work is in progress to try to improve the applicability of this device to a higher range of energy values of the particles to be analyzed and to carry out measurements in other laboratories with different lasers at different intensities and wavelengths.

\section{ACKNOWLEDGMENTS}

This work was supported by the "Research and Mobility" project of the Messina University (Italy) No. 74893496, coordinated by Professor L. Torrisi.

[1] L. Torrisi, D. Mascali, R. Miracoli, S. Gammino, N. Gambino, L. Giuffrida, and D. Margarone, Measurements of electron energy distribution in tantalum laser-generated plasma, J. Appl. Phys. 107, 123303 (2010).

[2] L. Torrisi, Coulomb-Boltzmann-shifted distribution in laser-generated plasmas from $10^{10}$ up to $10^{19} \mathrm{~W} / \mathrm{cm}^{2}$ intensities, Radiat. Eff. Defects Solids 171, 34 (2016).

[3] A. Macchi, M. Borghesi, and M. Passoni, Ion acceleration by superintense laser-plasma interaction, Rev. Mod. Phys. 85, 751 (2013).

[4] S. A. Gaillard et al., Increased laser-accelerated proton energies via direct laser-light-pressure acceleration of electrons in microcone targets, Phys. Plasmas 18, 056710 (2011).

[5] J. Braenzel, A. A. Andreev, K. Platonov, M. Klingsporn, L. Ehrentraut, W. Sandner, and M. Schnürer, Coulomb-driven energy boost of heavy ions for laser-plasma acceleration, Phys. Rev. Lett. 114, 124801 (2015).

[6] X. T. Wang, B. Y. Man, G. T. Wang, Z. Zhao, Y. Liao, B. Z. Xu, Y. Y. Xia, L. M. Mei, and X. Y. Hu, Optical spectroscopy of plasma produced by laser ablation of Ti alloy in air, J. Appl. Phys. 80, 1783 (1996).

[7] G. Bertuccio, D. Puglisi, L. Torrisi, and C. Lanzieri, Silicon carbide detector for laser-generated plasma radiation, Appl. Surf. Sci. 272, 128 (2013).

[8] L. Torrisi, S. Gammino, A. M. Mezzasalma et al., Laser ablation of UHMWPE-polyethylene by $438 \mathrm{~nm}$ high energy pulsed laser, Appl. Surf. Sci. 227, 164 (2004).

[9] L. Torrisi, M. Cutroneo, L. Andò, and J. Ullschmied, Thomson parabola spectrometry for gold laser-generated plasmas, Phys. Plasmas 20, 023106 (2013).

[10] G. A. P. Cirrone, A. Tramontana, G. Candiano et al., A new Thomson spectrometer for high energy laser-driven beams diagnostic, J. Instrum. 9, T08001 (2014).

[11] M. Cutroneo, L. Torrisi, L. Calcagno, and A. Torrisi, Thomson parabola spectrometry of laser generated plasma at PALS laboratory, J. Phys. Conf. Ser. 508, 012012 (2014).

[12] A. Alejo, S. Kar, A. Tebartz et al., High resolution Thomson parabola spectrometer for full spectral capture of multispecies ion beams, Rev. Sci. Instrum. 87, 083304 (2016).

[13] L. Torrisi and G. Costa, Magnetic focusing of emitted ions from laser-generated plasma: Enhancement of yield and energy, Laser Part. Beams 35, 202 (2017).

[14] L. Torrisi, G. Costa, G. Ceccio, A. Cannavò, N. Restuccia, M. Cutroneo, L. Torrisi, and M. Cutroneo, Magnetic and electric deflector spectrometers for ion emission analysis from laser generated plasma, EPJ Web Conf. 167, 03011 (2018).

[15] L. Torrisi, L. Giuffrida, M. Rosinski, and C. Schallhorn, Ge and $\mathrm{Ti}$ post-ion acceleration from laser ion source, Nucl. Instrum. Methods Phys. Res., Sect. B 268, 2808 (2010).

[16] A. Szydlowski, J. Badziak, P. Parys, J. Wolowski, E. Woryna, K. Jungwirth, B. Kralikova, J. Krasa, L. Laska, M. Pfeifer, K. Rohlena, J. Skala, J. Ullschmied, F. D. Boody, S. Gammino, and L. Torrisi, Measurements of energetic ions emitted from laser produced plasma by means of solid state nuclear tack detectors of the PM-355 type, Plasma Phys. Controlled Fusion 45, 1417 (2003).

[17] COMSOL (2019) Multiphysics Simulation SoftwareCOMSOL actual website, https://www.comsol.it/.

[18] G. Costa and L. Torrisi, Diagnostics of particles emitted from a laser generated plasma: Experimental data and simulations, EPJ Web Conf. 167, 04005 (2018).

[19] L. Torrisi, L. Andò, G. Ciavola, S. Gammino, and A. Barnà, Angular distribution of ejected atoms from Nd:YAG laser irradiating metals, Rev. Sci. Instrum. 72, 68 (2001).

[20] International specialty products, Gafchromic dosimetry media, type HD-810, actual website 2019: http://www .elimpex.com/new/products/radiation_therapy/ Gafchromic/content/GAFCHROMIC\%20HD-810.pdf.

[21] L. Torrisi, M. Cutroneo, and J. Ullschmied, TNSA and ponderomotive plasma production in enriched carbon polyethylene foils, Phys. Plasmas 24, 043112 (2017).

[22] J. F. Ziegler, M. D. Ziegler, and J. P. Biersack, SRIM-The stopping and range of ions in matter (2010), Nucl. Instrum. Methods Phys. Res., Sect. B 268, 1818 (2010).

[23] E. Woryna, P. Parys, J. Wołowski, and W. Mroz, Corpuscular diagnostics and processing methods applied in investigations of laser produced plasma as a source of highly ionized ions, Laser Part. Beams 14, 293 (1996).

[24] L. Torrisi and G. Costa, Simple magnetic spectrometer for ions emitted from laser-generated plasma at $10^{10} \mathrm{~W} / \mathrm{cm}^{2}$ intensity, Laser Part. Beams 36, 163 (2018).

[25] D. C. Carroll, P. Brummitt, D. Neely, F. Lindau, O. Lundh, C.-G. Wahlström, and P. McKenna, A modified Thomson parabola spectrometer for high resolution multi-MeV ion measurements-Application to laser-driven ion acceleration, Nucl. Instrum. Methods Phys. Res., Sect. A 620, 23 (2010).

[26] V. Malka, J. Faure, J. R. Marquès, F. Amiranoff, J. P. Rousseau, S. Ranc, J. P. Chambaret, Z. Najmudin, B. Walton, P. Mora, and A. Solodov, Characterization of electron beams produced by ultrashort (30 fs) laser pulses, Phys. Plasmas 8, 2605 (2001). 